\title{
EXACT AND APPROXIMATE COMPRESSION OF TRANSFER MATRICES FOR GRAPH HOMOMORPHISMS
}

\author{
PER HÅKAN LUNDOW AND KLAS MARKSTRÖM
}

\begin{abstract}
The aim of this paper is to extend the previous work on transfer matrix compression in the case of graph homomorphisms. For $H$-homomorphisms of lattice-like graphs we demonstrate how the automorphisms of $H$, as well as those of the underlying lattice, can be used to reduce the size of the relevant transfer matrices. As applications of this method we give currently best known bounds for the number of 4 - and 5-colourings of the square grid, and the number of 3- and 4-colourings of the threedimensional cubic lattice. Finally, we also discuss approximate compression of transfer matrices.
\end{abstract}

\section{Introduction}

Transfer matrices are a standard tool in various branches of mathematics. In enumerative combinatorics they have been used for a long time to solve counting problems which can be described using graph homomorphisms. In statistical physics transfer matrices have been a well used tool for the computation of partition functions of various spin models. In ergodic theory transfer matrices are used to describe the behaviour of a class of dynamical system known as subshifts of finite type. Recently the many similarities between these uses have been put on a firm mathematical ground. That the calculation of entropies for $\mathbb{Z}^{d}$-subshifts of finite type are equivalent to counting graph homomorphisms from $\mathbb{Z}^{d}$ into some graph $H$ was demonstrated in $[7]$. In $[\mathbf{6}]$ it was proved that counting weighted graph homomorphisms is equivalent to computation of the partition function for statistical physics models satisfying a condition known as reflection positivity. The study of phase transitions in statistical physics models has also begun to be studied in the language of homomorphisms [3].

A general limitation for all these applications is that transfer matrices tend to grow fast with the size of the system considered, thus limiting the size of the system one can work with. A recent development in this area is the use of automorphisms of the underlying graphs to reduce the size of the matrices. This was done for a special case in $[\mathbf{5}]$ and was developed as a general method in [13]. Our aim here is first to show how this method, now called transfer matrix compression, in many cases can be taken even further than earlier applications, and also to discuss how one can make even greater size reductions if one is ready to settle for bounds of the computed entropies rather than exact values. For many applications the latter is sufficient.

Received 23 February 2006, revised 17 August 2007; published 7 February 2008. 2000 Mathematics Subject Classification 05C99, 82B20.

(c) 2008, Per Håkan Lundow and Klas Markström 
Let us put things on a firmer ground. A homomorphism $\phi$ from a graph $G$ to a graph $H$, which may have loops, is a mapping which preserves adjacencies, i.e. if $(x, y) \in E(G)$ then $(\phi(x), \phi(y)) \in E(H)$. The set of all homomorphisms from $G$ to $H$ is denoted $\operatorname{Hom}(G, H)$. We say that $H$ is a weighted graph if there are two functions $\alpha_{H}: V(H) \rightarrow F$ and $\beta_{H}: E(H) \rightarrow F$, where $F$ is a ring. Given a weighted graph $H$ we assign a weight $w(\phi)$ to each homomorphism $\phi$ from $G$ to $H$

$$
w(\phi)=\prod_{x \in V(G)} \alpha_{H}(\phi(x)) \prod_{x y \in E(G)} \beta_{H}(\phi(x), \phi(y)) .
$$

Let us give a few examples:

EXAMPLE 1.1.

1. $H=K_{q}, \alpha=\beta=1$, corresponds to ordinary proper $q$-colourings.

2. Let $H$ be a $K_{2}$ with a loop on one vertex, let the edge have weight 1 , the loop weight $t$ and $\alpha=1$. For $t=1$ the homomorphisms correspond to independent sets and for general $t$, we have the so-called hard-core lattice gas model.

3. If $H$ is a $K_{q}$ with loops on every vertex and the weights are $t^{-1}$ on the loops, $t$ on the ordinary edges and 1 on the vertices, we have the $q$-state Potts model.

4. If we take the previous example with $q=2$ and put a weight $s$ on one vertex of $H$ and $s^{-1}$ on the other vertex, we have the Ising model with an external field.

If we have a model where we also want to put colours or 'spins' on the edges of $G$ (for example, when considering matchings, or on both edges and vertices), we can instead consider the line-graph or total-graph of the underlying graph $G$ respectively. It is also straightforward to generalise these concepts to hypergraphs if one wants to consider interaction between larger sets of vertices.

We next define a weighted counter for these homomorphisms

$$
Z(G, H)=\sum_{\phi \in \operatorname{Hom}(G, H)} w(\phi) .
$$

If all weights on $H$ are just 1 this will be exactly $|\operatorname{Hom}(G, H)|$. In spin models the weights are often taken to be of the form $e^{K}$ for a parameter $K$ which is interpreted as a temperature, and then $Z(G, H)$ is called the partition function of the model. In ergodic theory $Z(G, H)$ is called the pressure of the subshift described by $G$ and $H$. In most applications the aim is either to compute $Z(G, H)$ when $F$ is a ring of polynomials, as in $[\mathbf{9}]$, or to determine how fast $Z\left(G_{n}, H\right)$ grows when $F=\mathbb{R}$ and $G_{n}$ is some sequence of graphs; see, for example, $[\mathbf{7}, 5]$.

\section{Polygraphs and transfer matrices}

Transfer matrices are most useful for computation within a class of graphs known as polygraphs. This class was introduced in [1], where the transfer matrices were used to compute matching polynomials. A polygraph $\mathcal{G}$ is defined by a set of disjoint graphs $G_{1}, G_{2}, \ldots, G_{m}$ and a set of binary relations $\Upsilon_{1}, \Upsilon_{2}, \ldots, \Upsilon_{m}$, where $\Upsilon_{i} \subset$ $V\left(G_{i}\right) \times V\left(G_{i+1}\right)$. The vertex set of $\mathcal{G}$ is $\bigcup_{i} V\left(G_{i}\right)$ and the edge set is

$$
E(\mathcal{G})=\bigcup_{i} E\left(G_{i}\right) \bigcup_{i} \Upsilon_{i}
$$


If all $G_{i}=G$ and $\Upsilon_{i}=\Upsilon$ for all $i=1, \ldots, m$ we write the corresponding polygraph as $\mathcal{G}(G, \Upsilon, m)$.

Given a polygraph $\mathcal{G}$ and a weighted graph $H$ we can compute $Z(\mathcal{G}, H)$ using a sequence of transfer matrices. We define a matrix $M(i)$ for going from $G_{i}$ to $G_{i+1}$ as follows.

Let $\Phi\left(G_{i}\right)$ denote the set of restrictions of all homomorphisms in $\operatorname{Hom}(\mathcal{G}, H)$ to $G_{i}$. We call a member of $\Phi\left(G_{i}\right)$ a state on $G_{i}$. Now let the rows of $M(i)$ be indexed by the states on $G_{i}$ and the columns by the states on $G_{i+1}$. We set $M_{x, y}(i)=0$ if there is no homomorphism $\phi \in \operatorname{Hom}(\mathcal{G}, H)$ such that $\left.\phi\right|_{G_{i}}=x$ and $\left.\phi\right|_{G_{i+1}}=y$. If there exists such a $\phi$ we set $M_{x, y}$ equal to the contribution to the weight $w(\phi)$ of the edges in $\Upsilon_{i}$ and the edges and vertices of $G_{i+1}$. We also define an associated vector $\eta$. The position in $\eta$ corresponding to the row $x$ is set equal to the weight of the partial homomorphism $x$.

The partition function is now given by

$$
Z(\mathcal{G}, H)=\eta\left(\prod_{i} M(i)\right) \mathbf{1} .
$$

One can also consider cyclic polygraphs where the last relation $\Upsilon$ connects $G_{m}$ to $G_{1}$. In this case the partition function is given by the trace of the transfer matrix product,

$$
Z(\mathcal{G}, H)=\operatorname{Tr}\left(\prod_{i} M(i)\right) .
$$

\section{Exact compression of transfer matrices}

Henceforth we will assume that our polygraphs are on the form $\mathcal{G}(G, \Upsilon, m)$. Most of what follows can be adapted to general polygraphs as well. Let us recall that given an $N \times N$ matrix $M$ a partition $\mathcal{X}=\left\{X_{1}, X_{2}, \ldots, X_{r}\right\}$ of $\{1, \ldots, N\}$ is called an equitable partition if $\sum_{l \in X_{j}} M\left(i_{1}, j\right)=\sum_{l \in X_{j}} M\left(i_{2}, j\right)$ when $i_{1}, i_{2} \in X_{i}$.

Given a partition $X$ of the states on $G$, we define the compressed transfer matrix for $Z(\mathcal{G}, H)$ to be

$$
C_{\mathcal{X}}(i, j)=\sum_{l \in X_{j}} M(k, l), \quad k \in X_{i}, i, j=1 \ldots r .
$$

The main theorem of [13] can be stated as follows.

THEOREM 3.1. If $X$ is an equitable partition of $M$, then

$$
C_{\mathcal{X}}^{n}(i, j)=\sum_{l \in X_{j}} M^{n}(k, l), \quad k \in X_{i}, i, j=1 \ldots r
$$

This has the following corollary.

Corollary 3.2. Let $\eta$ be the vector of length $N$ whose ith entry is $\left|X_{i}\right| w\left(x_{i}\right)$, where $x_{i} \in X_{i}$. Then

$$
Z(\mathcal{G}, H)=\eta\left(\prod_{i} M(i)\right) \mathbf{1} .
$$


The main consequence of these results, which has been used in $[\mathbf{1 3}, \mathbf{5}, \mathbf{7}]$, and $[15,10,11,4]$ is as follows.

Corollary 3.3. If $M$ is a transfer matrix and the partition $\mathcal{X}$ consists of orbits on the set of states on $G$ under the automorphism group $\operatorname{Aut}(G)$, then $\mathcal{X}$ is equitable.

This corollary lets us make use of automorphisms of $G$ to compress our transfer matrices and for graphs with reasonably large automorphism group, such as cycles, the reduction in size can be substantial.

ExAmPLE 3.4. Let us look at the transfer matrix for $\operatorname{Hom}\left(G\left(P_{3}, \mathrm{Id}, n\right), K_{3}\right)$, that is, 3-colourings of the graph $P_{3} \times P_{n}$.

There are twelve states on $P_{3}$ and the only nontrivial member of $\operatorname{Aut}\left(P_{3}\right)$ is a reflection in the midpoint. If we use $1,2,3$ to denote colours, we find that there are nine orbits:

$$
\{\{121\},\{212\},\{313\},\{131\},\{232\},\{323\},\{123,321\},\{132,231\},\{213,312\}\} .
$$

Here we get the following $9 \times 9$ matrix, instead of a $12 \times 12$ matrix,

$$
C=\left[\begin{array}{lllllllll}
0 & 0 & 1 & 1 & 1 & 0 & 0 & 0 & 2 \\
0 & 0 & 1 & 0 & 1 & 1 & 0 & 0 & 2 \\
1 & 1 & 0 & 0 & 0 & 1 & 2 & 0 & 0 \\
1 & 0 & 0 & 0 & 1 & 1 & 2 & 0 & 0 \\
1 & 1 & 0 & 1 & 0 & 0 & 0 & 2 & 0 \\
0 & 1 & 1 & 1 & 0 & 0 & 0 & 2 & 0 \\
0 & 0 & 1 & 1 & 0 & 0 & 0 & 1 & 1 \\
0 & 0 & 0 & 0 & 1 & 1 & 1 & 0 & 1 \\
1 & 1 & 0 & 0 & 0 & 0 & 1 & 1 & 0
\end{array}\right], \quad \eta=\{1,1,1,1,1,1,2,2,2\}
$$

Even though we have reduced the side of the matrix by one quarter, we still have a fairly sparse matrix. The fact that we still have a sparse compressed matrix can on one hand be viewed a sign that the matrix could be compressed further to a dense matrix, as we will soon do, but for some cases it could also be taken advantage of by instead changing to computational techniques optimized for sparse matrices.

From algebraic graph theory [8] we know that if $G$ is any graph and $X$ is a partition of its vertices into orbits under $\operatorname{Aut}(G)$ then the corresponding partition is equitable. If we choose to interpret the transfer matrix $M$ as the adjacency matrix of a weighted graph, then what we did in Corollary 3.3 can be interpreted as using the subgroup of $\operatorname{Aut}(M)$ which is induced by $\operatorname{Aut}(G)$ to partition $M$. However, $\operatorname{Aut}(M)$ has an even larger subgroup induced by $\operatorname{Aut}(G) \times \operatorname{Aut}(H)$. Here $\operatorname{Aut}(H)$ is assumed to preserve the weights on $H$ as well as the adjacencies.

Corollary 3.5. Let $X$ be a partition of $M$ given by the orbits of $\operatorname{Aut}(G) \times \operatorname{Aut}(H)$; then $X$ is equitable.

When the graph $H$ is highly symmetric, such as in the case of proper colourings of $G$ or the partition function of the Potts model, the extra reduction in size achieved here can be remarkable.

EXAMPLE 3.6. If we use the automorphism group of $K_{3}$ as well in Example 3.4, we now only find two orbits,

$$
\{\{121,212,313,131,232,323\},\{123,321,132,231,213,312\}\},
$$


and the compressed transfer matrix has side 2 :

$$
C=\left[\begin{array}{ll}
3 & 2 \\
2 & 2
\end{array}\right], \quad v=\{6,6\} .
$$

\section{Application to the asymptotic number of q-colourings of lattices}

The number of proper $q$-colourings of an $n \times n$ square grid, or $P_{n} \times P_{n}$, is known to grow exponentially as a function of $n^{2}$. We denote the basis for this exponential growth by $\lambda^{s}(q)$. This is a quantity which is of interest both in enumerative combinatorics and statistical physics. In the latter case, $\log \lambda^{s}(q)$ is seen as the ground state entropy of the much studied antiferromagnetic Potts model on the square grid; see, for example, [16] for a survey.

For 3-colourings Lieb [12] found the exact asymptotic value of $\lambda^{s}(3)=\left(\frac{4}{3}\right)^{3 / 2}$ but for larger $q$ the value of $\lambda^{s}(q)$ is still unknown. More recently the antiferromagnetic Potts model has been studied, using transfer matrix and compression thereof, both in a series of papers by Jacobsen, Salas and Sokal $[\mathbf{1 5}, \mathbf{1 0}, \mathbf{1 1}]$, as well as in a collection of papers by Chang and Shrock; see, for example, [4].

As a full-scale example of our methods we now look at the compression of transfer matrices for $q$-colourings of the square grid for $q=4,5$, for the cubic grid $P_{n} \times$ $P_{n} \times P_{n}$ for $q=3,4$, and their use in getting bounds for $\lambda^{s}(q)$.

It is known that the maximum eigenvalues $\theta_{1}(k)$ and $\theta_{2}(k)$ of the transfer matrices for $\operatorname{Hom}\left(G\left(P_{k}, \mathrm{Id}, n\right), K_{q}\right)$ and $\operatorname{Hom}\left(G\left(C_{k}, \operatorname{Id}, n\right), K_{q}\right)$ respectively, can be used to give upper and lower bounds for $\lambda^{s}(q)$; see, for example, [7] for a general treatment. In particular

$$
\frac{\theta_{1}(k+1)}{\theta_{1}(k)} \leqslant \lambda^{s}(q) \leqslant \theta_{2}(2 k)^{1 / 2 k} .
$$

So we can bound $\lambda^{s}(q)$ by computing $\theta_{1}(k)$ for consecutive $k$ and $\theta_{2}(k)$ for even $k$.

In Table 1 we have given the size of the transfer matrix for $\operatorname{Hom}\left(G\left(P_{k}, \mathrm{Id}, n\right), K_{3}\right)$ and $\operatorname{Hom}\left(G\left(C_{k}, \mathrm{Id}, n\right), K_{3}\right)$. Here $N_{1}$ denotes the side of the uncompressed transfer matrix, $N_{2}$ the side when the automorphism groups of $P_{k}$ and $C_{k}$ respectively were used, and $N_{3}$ the size when the automorphism group of $K_{3}$ was used as well. The effect of the larger automorphism group of the cycle is easily visible, as is the gain from including the automorphism group of $K_{4}$ in the compression step. We can find $N_{1}$ exactly by evaluating the chromatic polynomial; see, for example, [14], of the cycle and the path, giving us $N_{1}=3^{k}+(-1)^{k} 3$ and $N_{1}=43^{k-1}$ for the cycle and paths respectively. For both the path and the cycle, $N_{1}$ grows with $\mathcal{O}\left(3^{k}\right)$. A typical colouring of, for example, the cycle is not invariant under any non-trivial automorphisms so we expect $N_{2}$ to be $\mathcal{O}\left(3^{n} /\left|\operatorname{Aut}\left(C_{k}\right)\right|\right)=\mathcal{O}\left(3^{k} / 2 k\right)$ and $N_{3}$ to be almost a factor 4 ! smaller, and for larger $k$ this is indeed close to what we see in Table 1.

Here we can also note an additional property that will hold for larger values of $q$, as well as for homomorphisms to other highly symmetrical graphs $H$. A $q$-colouring of a graph $G$ is equivalent to a partition of the vertex set of $G$ into independent sets together with an assignment of distinct colours to the parts of this partition. When an element $\sigma \in \operatorname{Aut}(G) \times \operatorname{Aut}(H)$ acts on a colouring, or homomorphism in general, the part of $\sigma$ from $\operatorname{Aut}(G)$ acts on the parts of the partition by moving 
them on the graph, and the part of $\sigma$ from $\operatorname{Aut}(H)$ acts by changing the labels of the parts. When $q$ is larger than the order of $G$, the number of parts in a partition of $V(G)$ must be smaller than $q$, and as $q$ increases $N_{1}$ can only increase by increasing the number of possible labelings of the parts of the partitions. This means that while $N_{1}$ will continue to grow with $q$, for a fixed $G$, the value of $N_{3}$ will not. This observation has been used in [15], where $q$-colourings for large values of $q$ were studied. Using this observation, one can also bypass the step of computing the full set of colourings corresponding to $N_{1}$ and instead start out with partitions of the vertex set of $G$, but in the current paper we will stay with the basic method of starting out with all colourings of $G$.

For small $k$, the transfer matrices and eigenvalues were computed first with a Mathematica program and also with a Fortran 90 program. For larger $k$ the ForTRAn 90 program was run on a Linux cluster. In Table 2 of Appendix A we have collected the computed eigenvalues. The higher-precision values for small $k$ are due to the MATHEMATiCA program.

Using these eigenvalues and inequalities (6) we find the following bounds for $\lambda^{s}(4)$ :

$$
2.336056640723116 \leqslant \lambda^{s}(4) \leqslant 2.33606820555777 .
$$

To our knowledge these are currently the best rigorous bounds for $\lambda^{s}(4)$. In $[2]$ the first terms of a series expansion in $1 /(q-1)$ for $\lambda^{s}(q)$ was obtained, and using this series it was estimated that

$$
\lambda^{s}(4)=2.336056641 \pm 0.000000001,
$$

with a heuristic error bound, an estimate which fits in just above our lower bound.

In the same way we computed the corresponding eigenvalues for 5-colourings, given in Table 3 of Appendix A. The bounds so obtained for $\lambda^{s}(5)$ are

$$
3.2504049231640764 \leqslant \lambda^{s}(5) \leqslant 3.250407145038276 .
$$

For the growth rate $\lambda^{c}(q)$ of the number $q$-colourings of the cubic lattice $P_{k} \times$ $P_{k} \times P_{k}$ there are no exact results known. In [2] series estimates were also given for $\lambda^{c}(3)$ and $\lambda^{c}(4)$, however these estimates were based on much shorter series than

Table 1: Transfer matrix sizes for 4-colourings. The upper three rows are for transferring a path, and the lower three for a cycle.

\begin{tabular}{cccccccccc}
\hline$n$ & 3 & 4 & 5 & 6 & 7 & 8 & 9 & 10 & 11 \\
\hline$N_{1}$ & 36 & 108 & 324 & 972 & 2916 & 8748 & 26244 & 78732 & 236196 \\
$N_{2}$ & 24 & 54 & 180 & 486 & 1512 & 4374 & 13284 & 39366 & 118584 \\
$N_{3}$ & 2 & 4 & 10 & 25 & 70 & 196 & 574 & 1681 & 5002 \\
\hline$N_{1}$ & 24 & 84 & 240 & 732 & 2184 & 6564 & 19680 & 59052 & 177144 \\
$N_{2}$ & 4 & 21 & 24 & 92 & 156 & 498 & 1096 & 3210 & 8052 \\
$N_{3}$ & 1 & 3 & 2 & 9 & 10 & 34 & 57 & 169 & 366 \\
\hline
\end{tabular}


those for the square grid and were given as

$$
\begin{gathered}
\lambda^{c}(3)=1.4435 \pm 0.0005 \\
\lambda^{c}(4)=2.043 \pm 0.001
\end{gathered}
$$

In order to compute bounds for the cubic lattice we can make use of the observations that $\lambda^{c}(q)$ is greater than the maximum eigenvalue for $\operatorname{Hom}\left(G\left(P_{k} \times\right.\right.$ $C_{\ell}$, Id, $\left.n\right), K_{q}$ ), since colourings of these graphs can be extended to periodic colourings of $\operatorname{Hom}\left(G\left(P_{k} \times P_{\ell}, \mathrm{Id}, n\right), K_{q}\right)$ for all $t$. Likewise, $\lambda^{c}(q)$ is less than the maximum eigenvalue for $\operatorname{Hom}\left(G\left(P_{k} \times P_{\ell}, \mathrm{Id}, n\right), K_{q}\right)$, since the number of colourings is submultiplicative.

As before, we can get lower bounds for the maximum eigenvalue of $\operatorname{Hom}\left(G\left(P_{k} \times\right.\right.$ $\left.C_{\ell}, \mathrm{Id}, n\right), K_{q}$ ) for a fixed $\ell$ by computing the maximum eigenvalues for consecutive $k$, and for each value of $\ell$ we will get a lower bound for $\lambda^{c}(q)$. Similarly we can get upper bounds for the maximum eigenvalue of $\operatorname{Hom}\left(G\left(P_{k} \times P_{\ell}, \operatorname{Id}, n\right), K_{q}\right)$ by computing the maximum eigenvalue of $\operatorname{Hom}\left(G\left(C_{k} \times P_{\ell}, \mathrm{Id}, n\right), K_{3}\right)$ for even $k$. These eigenvalues are in turn bounded from above by the maximum eigenvalue of $\operatorname{Hom}\left(G\left(C_{k} \times C_{\ell}, \mathrm{Id}, n\right), K_{3}\right)$, for even $k$ and $\ell$.

For $\lambda^{c}(3)$ our best bounds come from the eigenvalues of $\operatorname{Hom}\left(G\left(C_{6} \times C_{6}, \operatorname{Id}, n\right), K_{q}\right)$ and $\operatorname{Hom}\left(G\left(P_{k} \times C_{4}, \operatorname{Id}, n\right), K_{q}\right)$; for $\lambda^{c}(4)$ the bounds were achieved by $\operatorname{Hom}\left(G\left(C_{4} \times\right.\right.$ $\left.\left.C_{4}, \mathrm{Id}, n\right), K_{q}\right)$ and $\operatorname{Hom}\left(G\left(P_{k} \times C_{4}, \mathrm{Id}, n\right), K_{q}\right)$ :

$$
\begin{aligned}
& 1.4460096817417 \leqslant \lambda^{c}(3) \leqslant 1.4470681274660 ; \\
& 2.0343787307189 \leqslant \lambda^{c}(4) \leqslant 2.0652128520667 .
\end{aligned}
$$

As we can see, the estimate from [2] for $\lambda^{c}(4)$ is within our bounds but their estimate for $\lambda^{c}(3)$ is well below the lower bound, even when their heuristic error estimate is taken into account. As mentioned in $[2]$ this kind of "miss" by the series estimate could indicate a physically interesting structure in the set of 3-colourings.

\section{Approximate compression}

For many applications, the $\operatorname{ring} F$ is the real numbers and one typically has only positive weights. If the aim is to compute only the maximum eigenvalue of $M$, as in Example 4, we can go further with our compression than in the previous section. First we here only need to care about the so-called 'main part' of the spectrum of $M$, that is, the eigenvalues with eigenvector not orthogonal to $\mathbf{1}$, and of the main part we need only to preserve the maximum eigenvalue. We can now make use of one of the standard theorems of spectral graph theory; see, for example, [8].

THEOREM 5.1 (Interlacing of eigenvalues). Let $S$ be an $n \times m$ matrix such that $\left.S^{T} S\right)=I$ and $M$ a hermitian $n \times n$ matrix, and set $M^{\prime}=S^{T} M S$. Let the eigenvalues of $M$ be $\lambda_{1} \geqslant \lambda_{2}, \ldots, \lambda_{n}$ and those of $M^{\prime}$ be $\theta_{1} \geqslant \theta_{2}, \ldots, \geqslant \theta_{m}$. Then the eigenvalues of $M^{\prime}$ interlace the eigenvalues of $M$; that is,

$$
\lambda_{j} \geqslant \theta_{j} \geqslant \lambda_{n-m+j} .
$$

Corollary 5.2. Let $M$ be an $m \times m$ hermitian matrix and let $X=\left\{X_{1}, X_{2}, \ldots\right\}$ be a partition of $\{1, \ldots, m\}$. Define a matrix $B$ where $B_{i, j}$ is the average row sum in $M_{X_{i}, X_{j}}$. Then the eigenvalues of $B$ interlace those of $M$. 
Proof. Apply Theorem 5.1 with the matrix $S$ given by $S_{i, j}=\left|X_{i}\right|^{-1 / 2}$ if $j \in X_{i}$ and 0 elsewhere.

For a partition which is not necessarily equitable we thus find the following corollary.

Corollary 5.3. Let $\mathcal{X}$ be a partition of the rows and columns of $M$; then the maximum eigenvalue of $C(\mathcal{X})$ gives a lower bound on $\lambda_{1}(M)$.

Given a partition $\mathcal{X}$, we can also define a matrix $D$, defined as in the previous corollary but using the maximum row sum rather than the average.

Corollary 5.4. The maximum eigenvalue of $D$ gives an upper bound on $\lambda_{1}(M)$.

For many choices of weighted graph $H$ it is the case that $Z(G, H)$ is either subor super-additive with respect to the addition of edges and/or vertices to $G$. In this situation the corollaries of the interlacing theorem can be used to give us upper and lower bounds on the asymptotics of the maximum eigenvalues as $G$ becomes larger. These bounds can then in turn be used in combination with inequalities like (6).

Apart from getting bounds on the eigenvalues of $M$, one can also use an approximately compressed transfer matrix to speed up iterative algorithms for computing the exact maximum eigenvalue of $M$. Many such algorithms, such as the basic Perron-iteration, start out with a vector $v_{0}$, and the number of iterations in the algorithms depends on how close $v_{0}$ is to the eigenvector of the maximum eigenvalue. Using an approximately compressed transfer matrix, we can quickly find the maximum eigenvector of the compressed matrix; next this can vector can be expanded to a vector $v_{0}$ by replicating the $i$ th entry in the shorter vector in each of the $\left|X_{i}\right|$ entries correpsonding to that entry in the uncompressed vector.

In both Corollary 5.3 and Corollary 5.4 the choice of partition $\mathcal{X}$ will influence the value of the eigenvalue bound. How the partition should be chosen in order to get a good approximation will depend on the underlying graphs and weights, and it is hard to say anything much more precise than that one should strive to get blocks $M_{X_{i}, X_{j}}$ with as closely concentrated row-sums as possible.

EXAMPLE 5.5. In order to demonstrate the approximate bounds, and the influence of the choice of partition $\mathcal{X}$, we have computed these bounds for the transfer matrix for the number of 4-colourings on $P_{12} \times P_{n}$ and $C_{14} \times P_{n}$.

We have used two kinds of partition $\mathcal{X}$.

1. For the first type of partition we viewed each colouring as an integer written in base 4; for cycles we chose an arbitrary vertex to be the lowest digit. Next we sorted the colourings as if they were integers.

Given this sorted list of the colourings, we partitioned the list into consecutive sublists of length $k$ and $k-1$, with as few lists of length $k-1$ as possible.

2. As our second kind of partition we randomly partitioned the list of colours into parts of size $K$.

For these two partitions we next computed an upper bound for the largest eigenvalue of the transfer matrix for $C_{14}$ and a lower bound for that of $P_{12}$. For each graph and partitioning we chose $K$ so as to give compressed matrices with sides from 0.9 times the original side down to 0.1 . For the random ordering, we tried several random partitions. 


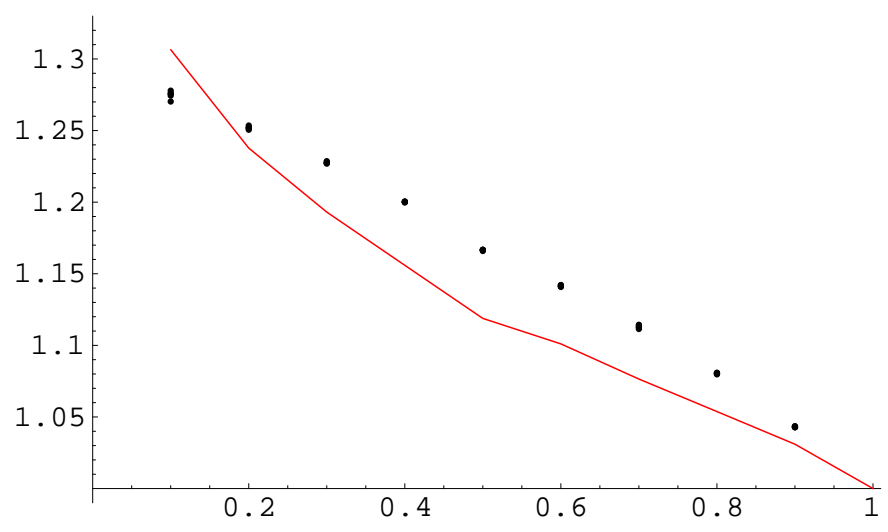

Figure 1: Approximate eigenvalues for $C_{14}$. Connected points are from the integer encoding. Clusters of isolated points are random partitions.

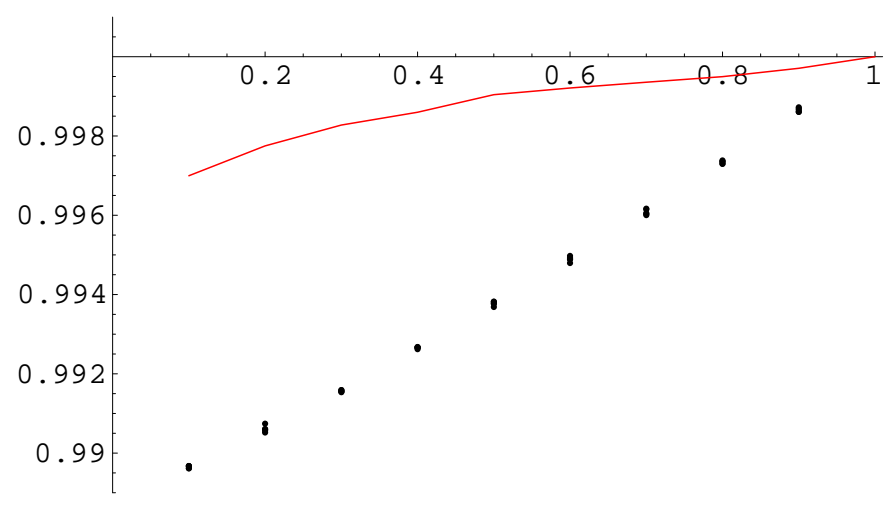

Figure 2: Approximate eigenvalues for $P_{12}$. Connected points are from the integer encoding. Clusters of isolated points are random partitions.

In Figures 1 and 2 we have plotted the approximate eigenvalue divided by the correct eigenvalue. We find that the lower bounds tend to be more accurate than the upper bounds. In both cases the integer encoding partition gives a noticeably better approximation than the random partitions. However, for the lower bound even the random partitions can be used to compress the matrix down to one tenth of its original side and still get a bound which is just one percent less than the correct value.

\section{Acknowledgements}

The computer resources needed for our computational work was provided by PDC, Stockholm, Sweden. The authors would like to thank the anonymous referee for constructive criticism. 
Appendix A. Colouring eigenvalues for the square grid

Table 2: Maximum eigenvalues for 4-colourings of the square grid

\begin{tabular}{rll}
\hline$k$ & $P_{k}$ & $C_{k}$ \\
\hline 3 & 16.34846922834953429459185 & 11 \\
4 & 38.18874899819785577572648 & 31.69693845669906858918370 \\
5 & 89.20972864650976523895547 & 67.01514803843835560759098 \\
6 & 208.3980964253975720633538 & 165.6008220944556672481883 \\
7 & 486.8291555413566000543864 & 375.4804004152886797996016 \\
8 & 1137.260058429224259797968 & 892.2418753486577354212783 \\
9 & 2656.703606588566986303095 & 2064.554606528212648447034 \\
10 & 6206.209878666071640432711 & 4849.504943339923099642784 \\
11 & 14498.05763470071954268293 & 11293.10916510243643781710 \\
12 & 33868.2836924334 & 26429.64958444568607749808 \\
13 & 79118.2289428612 & 61675.61597454731 \\
14 & 184824.664073982 & 144167.2612085567 \\
15 & 431760.883879445 & 336660.4085235824 \\
16 & & 786626.0015010700 \\
\hline
\end{tabular}

Table 3: Maximum eigenvalues for 5-colourings of the square grid

\begin{tabular}{rll}
\hline$n$ & $P_{n}$ & $C_{n}$ \\
\hline 2 & 13 & \\
3 & 42.254746265138 & 32 \\
4 & 137.34484848076 & 114.16796064692 \\
5 & 446.42629917197 & 359.90932515034 \\
6 & 1451.0662111085 & 1182.6934618883 \\
7 & 4716.5527439510 & 3829.1466667249 \\
8 & 15330.706253905 & 12464.383871815 \\
9 & 49831.003080926 & 40492.334305935 \\
10 & 161970.93773951 & 131643.39169572 \\
11 & 526471.13343803 & 427861.13442804 \\
12 & & 1390763.1270219 \\
\hline
\end{tabular}


Appendix B. Colouring eigenvalues for the cubic lattice

Table 4: Maximum eigenvalues for 3-colourings of $G \times P_{2}$

\begin{tabular}{rll}
\hline$n$ & $G=P_{n}$ & $G=C_{n}$ \\
\hline 3 & 13.8072589673052 & 3 \\
4 & 30.1109468160278 & 26.6214214843774 \\
5 & 65.8601729387350 & 24.6080079665097 \\
6 & 144.283083091960 & 123.199451464237 \\
7 & 316.392676802175 & 148.219336059660 \\
8 & 694.239161184508 & 582.950876259572 \\
9 & 1523.97594483322 & 806.285660630712 \\
10 & 3346.41981099416 & 2782.03759223168 \\
11 & 7349.89922843146 & 4196.30975248900 \\
\hline
\end{tabular}

Table 5: Maximum eigenvalues for 3-colourings of $G \times P_{3}$

\begin{tabular}{lll|}
\hline$n$ & $G=P_{n}$ & $G=C_{n}$ \\
\hline 3 & 42.9509955498558 & 4.56155281280883 \\
4 & 134.633390548866 & 114.548378741056 \\
4 & 423.398960388624 & 103.444398290072 \\
5 & 1333.80481197401 & 1091.43690942498 \\
6 & 4206.08745616625 & 1449.29878537714 \\
7 & & 10674.0945361673 \\
\hline
\end{tabular}

Table 6: Maximum eigenvalues for 3-colourings of $G \times P_{4}$

\begin{tabular}{lll}
\hline$n$ & $G=P_{n}$ & $G=C_{n}$ \\
\hline 4 & 607.5008342289296 & 496.9033949197111 \\
5 & 2751.292994653581 & 437.9397858090114 \\
6 & 12483.36568754961 & 9768.207310946096 \\
\hline
\end{tabular}

Table 7: Maximum eigenvalues for 3-colourings of $G \times P_{5}$

\begin{tabular}{lll}
\hline$n$ & $G=P_{n}$ & $G=C_{n}$ \\
\hline 5 & 17953.38896417563 & $1859.891162040439^{6}$ \\
\hline
\end{tabular}


Table 8: Maximum eigenvalues for 3-colourings of $G \times C_{3}$

\begin{tabular}{rll}
\hline$n$ & $G=P_{n}$ & $G=C_{n}$ \\
\hline 2 & 3 & \\
3 & 4.56155281280883 & 2 \\
4 & 6.97196076839709 & 6.37228132326901 \\
5 & 10.6828851212084 & 6 \\
6 & 16.3920411989578 & 14.5064314940480 \\
7 & 25.1740785316175 & 15.7833418763922 \\
8 & 38.6831608665319 & 33.6767869577220 \\
9 & 59.4651079147947 & 39.6505660120334 \\
10 & 91.4379622705829 & 78.8188645918277 \\
11 & 140.631735559805 & 97.5298788390751 \\
12 & 216.327079158290 & 185.239857806635 \\
13 & 332.808012753772 & 237.182998032027 \\
\hline
\end{tabular}

Table 9: Maximum eigenvalues for 3-colourings of $G \times C_{4}$

\begin{tabular}{lll}
\hline$n$ & $G=P_{n}$ & $G=C_{n}$ \\
\hline 2 & 26.62142148437744 & \\
3 & 114.5483787410569 & \\
4 & 496.9033949197111 & 420.477039628259 \\
5 & 2163.237391033718 & 378.843114768611 \\
6 & 9435.406059898469 & 7704.08921920854 \\
7 & & 11291.7201866529 \\
8 & & 144633.687249454 \\
\hline
\end{tabular}

Table 10: Maximum eigenvalues for 3-colourings of $G \times C_{5}$

\begin{tabular}{lll}
\hline$n$ & $G=P_{n}$ & $G=C_{n}$ \\
\hline 2 & 24.6080079665097 & \\
3 & 103.444398290072 & \\
4 & 437.939785809011 & \\
5 & 1859.89116204043 & 408.155175807023 \\
6 & 7912.06577168573 & 6610.84386549256 \\
\hline
\end{tabular}

Table 11: Maximum eigenvalues for 3-colourings of $G \times C_{6}$

\begin{tabular}{lll}
\hline$n$ & $G=P_{n}$ & $G=C_{n}$ \\
\hline 6 & & 599243.330687515 \\
\hline
\end{tabular}




\section{References}

1. Darko Babić, Ante Graovac, Bojan Mohar and Tomaž Pisanski, 'The matching polynomial of a polygraph', Discrete Appl. Math. 15 (1986) 11-24. 2

2. A. V. BakAev and V. I. Kabanovich, 'Series expansions for the $q$-colour problem on the square and cubic lattices', J. Phys. A 27 (1994) 6731-6739. 6,7

3. Graham R. Brightwell and Peter Winkler, 'Graph homomorphisms and phase transitions', J. Combin. Theory Ser. B 77 (1999) 221-262. 1

4. Shu-Chiuan Chang and Robert Shrock, 'Tutte polynomials and related asymptotic limiting functions for recursive families of graphs', Adv. in Appl. Math. 32, Special issue on the Tutte polynomial (2004) 44-87. 4, 5

5. M. Ciucu, 'An improved upper bound for the 3-dimensional dimer problem', Duke Math. J. 94 (1998) 1-11. 1, 2, 4

6. M. Freedman, L. Lovász and A. Schrijver, 'Reflection positivity, rank connectivity, and homomorphism of graphs', J. Amer. Math. Soc. 20 (2007) 37-51. 1

7. Shmuel Friedland and URi N. Peled, 'Theory of computation of multidimensional entropy with an application to the monomer-dimer problem', $A d v$. in Appl. Math. 34 (2005) 486-522. 1, 2, 4, 5

8. Chris Godsil and Gordon Royle, Algebraic graph theory, Graduate Texts in Mathematics 207 (Springer, New York, 2001). 4, 7

9. R. HÄGGKVIST and P. H. Lundow, 'The Ising partition function for 2D grids with cyclic boundary: computation and analysis', J. Statist. Phys. 108 (2002) 429-457. 2

10. Jesper LykKe Jacobsen and Jesús SAlAs, 'Transfer matrices and partition-function zeros for antiferromagnetic Potts models. II. Extended results for square-lattice chromatic polynomial', J. Statist. Phys. 104 (2001) 701-723. 4,5

11. Jesper Lykke Jacobsen, Jesús Salas and Alan D. Sokal, 'Transfer matrices and partition-function zeros for antiferromagnetic Potts models. III. Triangular-lattice chromatic polynomial', J. Statist. Phys. 112 (2003) 9211017. 4, 5

12. Elliott H. Lieb, 'Residual entropy of square ice', Phys. Rev. 162 (1967) $162-172.5$

13. PER HÅKAn Lundow, 'Compression of transfer matrices', 17th British Combinatorial Conference, Canterbury, 1999, Discrete Math. 231 (2001) 321-329. $1,3,4$

14. R. C. Read and W. T. Tutte, 'Chromatic polynomials', Selected topics in graph theory 3 (Academic Press, San Diego, CA, 1988) 15-42. 5

15. Jesús SAlas and Alan D. Sokal, 'Transfer matrices and partition-function zeros for antiferromagnetic Potts models. I. General theory and square-lattice chromatic polynomial.' J. Statist. Phys. 104 (2001) 609-699. 4, 5, 6

16. F. Y. Wu, 'The Potts model', Rev. Modern Phys. 54 (1982) 235-268. 5 
Per Håkan Lundow phl@kth.se

http://www. theophys.kth.se/ p phl

KTH Physics

AlbaNova University Center

SE-106 91 Stockholm

Sweden

Klas Markström Klas.Markstrom@math.umu.se

http://abel.math.umu.se/ Rlasm

Department of Mathematics and Mathematical Statistics

Umeå University

SE-901 87 Umeå

Sweden 